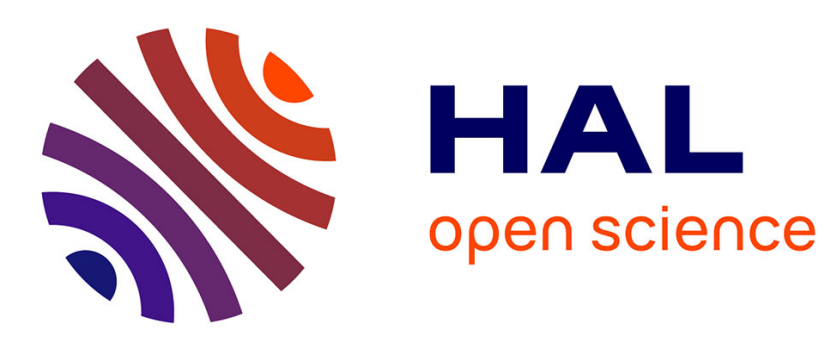

\title{
Un espace de conception fondé sur une analyse morphologique des techniques de menus
}

\author{
Mathieu Nancel, Michel Beaudouin-Lafon, Stéphane Huot
}

\section{To cite this version:}

Mathieu Nancel, Michel Beaudouin-Lafon, Stéphane Huot. Un espace de conception fondé sur une analyse morphologique des techniques de menus. IHM 2009, 21ème conférence francophone sur l'Interaction Homme-Machine, 2009, Grenoble, France. pp.ACM, International Conference Proceedings Series, 10.1145/1629826.1629829 . inria-00550594

\section{HAL Id: inria-00550594 https://hal.inria.fr/inria-00550594}

Submitted on 28 Dec 2010

HAL is a multi-disciplinary open access archive for the deposit and dissemination of scientific research documents, whether they are published or not. The documents may come from teaching and research institutions in France or abroad, or from public or private research centers.
L'archive ouverte pluridisciplinaire HAL, est destinée au dépôt et à la diffusion de documents scientifiques de niveau recherche, publiés ou non, émanant des établissements d'enseignement et de recherche français ou étrangers, des laboratoires publics ou privés. 


\section{Un espace de conception fondé sur une analyse morphologique des techniques de menus}

\author{
Mathieu Nancel ${ }^{1,2}$ \\ Stéphane Huot ${ }^{1,2}$ \\ ${ }^{1}$ LRI - Univ. Paris-Sud \& CNRS \\ Bât. 490, F-91405 Orsay, France
}

\author{
Michel Beaudouin-Lafon ${ }^{1,2}$ \\ ${ }^{2}$ INRIA \\ Bât. 490, F-91405 Orsay, France
}

\{nancel,huot,mbl\}@Iri.fr

\begin{abstract}
RESUME
Cet article présente un espace de conception basé sur une analyse morphologique des mécanismes de structuration des menus et de sélection des items. Son but est de faciliter l'exploration de nouveaux types de menus afin notamment d'augmenter leur capacité sans détériorer leurs performances. L'article démontre l'aspect génératif de cet espace de conception grâce à quatre nouveaux designs de menus, basés sur des combinaisons de dimensions pas ou peu explorées. Pour deux d'entre eux, des expérimentations contrôlées montrent qu'ils offrent des performances comparables aux menus de la littérature.
\end{abstract}

MOTS CLES : Menus, Analyse morphologique, Espace de conception

\section{ABSTRACT}

This paper presents a design space based on a morphological analysis of menu techniques. The goal of this design space is to facilitate the exploration of novel menu designs, in particular to increase menu capacity without sacrificing performance. The paper demonstrates the generative aspect of this design space with four new menu designs based on poorly explored combinations of input dimensions. For two of these four designs, the paper presents controlled experiments that show that they perform on a par with other menus from the literature.

CATEGORIES AND SUBJECT DESCRIPTORS: H.5.2 Information Systems : Information Interfaces and Presentation - User Interfaces, Input Devices and Strategies.

GENERAL TERMS: Human Factors

KEYWORDS: Menus, Morphological analysis, Design space

\section{INTRODUCTION}

Les menus sont probablement la technique d'interaction la plus utilisée dans les interfaces graphiques. De ce fait, ils ont fait l'objet de très nombreux travaux de recherche qui ont notamment conduit à la création d'un nombre considérable de techniques. Ce domaine connait un regain d'intérêt avec la multiplication des plateformes d'interaction telles que dispositifs mobiles, tables interactives, murs d'images, et des périphériques d'entrée : entrée tactile, au stylo, tangible, etc. Ces nouveaux contextes d'interaction remettent en cause les résultats acquis pour la situation classique écran-clavier-souris et justifient que des techniques adaptées soient développées et testées.

Cependant la profusion des techniques proposées et des évaluations présentées rend difficile le choix d'une technique pour un contexte donné. Il parait donc nécessaire d'organiser ce corpus de connaissance afin de mieux comprendre ce qui est acquis et de mieux identifier ce qu'il reste à explorer. Dans cet esprit, Bailly a récemment produit l'espace de conception MenUA [3] fondé sur les facteurs d'utilisabilité et d'applicabilité, eux-mêmes décomposés en critères. Le travail présenté dans cet article poursuit un objectif comparable mais est fondé sur la morphologie des techniques de menus.

La morphologie de l'interaction [10, 7] consiste à étudier les propriétés des techniques d'interaction afin d'identifier des dimensions d'analyse permettant de comprendre les différences et similitudes entre techniques existantes et de faciliter la création de nouvelles techniques. L'espace morphologique ainsi défini est donc à la fois analytique et génératif. Dans le travail présenté ici, la morphologie des techniques de menus est analysée selon deux points de vue : d'une part les dimensions d'entrée et la façon dont elles sont utilisées et combinées pour permettre la sélection des items; d'autre part la structuration des menus en groupes d'items et en sous-menus. Cette approche permet de prendre en compte notamment la diversité croissante des canaux d'entrée utilisés par les menus et la nécessité d'améliorer la capacité des menus et l'organisation des items. 
La suite de l'article présente un rapide état de l'art des menus et des espaces de conception de menus. Il décrit ensuite notre espace morphologique, puis illustre son pouvoir analytique. Enfin, il montre comment cet espace nous a permis d'imaginer quatre nouvelles techniques de menus, dont deux ont fait l'objet d'évaluations qui sont brièvement présentées : Dartboard et PushMenu. L'article se termine par quelques perspectives de travaux futurs.

\section{ETAT DE L'ART}

Un menu peut être défini comme une technique permettant une interaction de choix d'un item parmi un ensemble [14]. Cependant toutes les techniques de choix ne sont pas des menus, et il peut être difficile d'établir une frontière claire. Dans cet article nous appelons menu une technique qui permet de spécifier un item parmi un ensemble discret et stable de valeurs prédéfinies, éventuellement structuré de façon hiérarchique, et qui permet une visualisation transitoire, pendant l'interaction, des choix possibles. Cette définition exclut notamment les palettes et les boutons radio (affichage permanent des choix), les équivalents clavier (pas de visualisation possible des choix pendant l'interaction) et les listes (ensemble variable d'items).

Deux grandes catégories de menus sont les menus linéaires, présents dans toutes les interfaces actuelles, et les menus circulaires : Pie Menu [9], Marking Menu [26, 20, 21] et leurs nombreuses variantes, qui pour la plupart augmentent la capacité du Marking Menu à l'aide de dimensions supplémentaires. Par exemple, Hybrid Menu [16] et Flower Menu [6] sont deux techniques qui utilisent la courbure de la fin du geste pour augmenter le nombre d'items accessibles pour une même orientation donnée. Hybrid Menu atteint ainsi une capacité de 12 items et Flower Menu a une capacité théorique de 56 items.

Zhao et al. ont présenté les menus dits Tap-and-Stroke [28] qui combinent la position et l'orientation. Le principe est de désigner une position à l'écran, le tap, puis de dessiner une marque rectiligne, dont l'interprétation se fera en fonction de son orientation et de sa position par rapport au tap. Zhao et al. présentent deux techniques basées sur ce design, Polygon Menu et Zone Menu. Pressure Menu [25], quant à lui, combine l'orientation d'un trait effectué à l'aide d'un stylet et l'évolution de la pression durant l'exécution de ce trait. Quatre signatures de pression sont considérées en combinant une pression forte ou faible au début ou à la fin du geste, mais cette technique n'a pas été formellement évaluée.

L'un des principaux critères de conception de ces menus a été l'amélioration de la performance experte. Cela a conduit à des succès indéniables, comme le gain d'un facteur supérieur à 3 pour les Marking Menus, mais au détriment parfois d'autres caractéristiques, notamment la facilité d'apprentissage et la capacité des menus. Ainsi les Marking Menus sont limités à 8 items par niveau, et la difficulté d'apprentissage des Polygon Menus a été démontrée face aux Flower Menus [6]. Par contre, la question de la capacité des menus et de l'adéquation d'un ensemble d'items à une structure prédéfinie a été, à notre connaissance, peu ou pas étudiée.

D'autres travaux ont exploré l'usage de nouvelles dimensions d'entrée soit pour améliorer la performance, soit pour adapter le menu au contexte de l'interaction (cas des dispositifs mobiles et de l'interaction tactile), soit pour mieux intégrer les menus au reste de l'interface, notamment en réduisant les problèmes de changement de mode dans les interfaces à base de reconnaissance de marques. Multitouch Menu [4] exploite les avantages des tables tactiles en permettant à un utilisateur d'utiliser les cinq doigts de la main pour effectuer une sélection. Il combine un Marking Menu placé sous le pouce à des items positionnés sous chacun des doigts de la main, dont la position est repérée grâce à celle de la paume. Tilt Menu [27] utilise l'angle d'inclinaison du stylet pour spécifier une commande et ainsi permet de changer facilement de mode dans les interfaces à stylet.

Comme la performance experte a souvent été le critère principal d'évaluation des menus, une approche largement répandue consiste à utiliser les lois du mouvement humain, comme la loi de Fitts [13]pour modéliser le temps de réaction. Un bon exemple est le modèle prédictif de performance de Cockburn et al. [12]. Enfin des modèles plus généraux peuvent s'appliquer à l'analyse des menus, comme les modèles GOMS [11] et le modèle CIS [2].

Au total, malgré une littérature abondante et le développement de nombreuses techniques, l'étude des menus manque encore d'approches analytiques et génératives afin d'explorer plus systématiquement l'espace de conception. Les travaux de Bailly [3] sur l'espace MenUA font exception, avec une analyse détaillée et multicritères des techniques de menus du point de vue de leur utilisabilité et de leur applicabilité. L'approche que nous présentons dans cet article est différente puisque nous ne partons pas de critères externes comme dans MenuA, mais des caractéristiques morphologiques internes des menus et des techniques d'interaction. En cela, notre approche est inspirée des travaux de Card et al. [10] sur l'analyse morphologique de l'espace de conception des périphériques d'entrée, dont elle reprend l'idée d'analyser les dimensions physiques de l'interaction.

\section{ANALYSE MORPHOLOGIQUE DES TECHNIQUES DE MENUS}

La sélection d'un item parmi un ensemble peut être caractérisée d'une part par la cardinalité et la structure de l'ensemble considéré, d'autre part par la séquence d'interactions qui permet à l'utilisateur de spécifier son choix de façon univoque. Une technique de menu est d'autant plus flexible que la structure des items peut être fidèlement reflétée dans le menu choisi; une technique est d'au- 
tant plus efficace que l'interaction est rapide et naturelle. Une façon d'opérationnaliser, au moins en partie, ces deux notions est d'évaluer l'information échangée dans le système : d'une part, l'organisation et le contenu du menu fournit à l'utilisateur une certaine quantité d'information, d'autre part l'interaction de l'utilisateur fournit au système l'information nécessaire à spécifier son choix.

\section{Approche informationnelle}

Cet échange d'information met en évidence deux goulots d'étranglement potentiels : en sortie, la capacité pour le système de fournir l'information (affichage du menu) et pour l'utilisateur de la recevoir (perception des choix possibles); en entrée, la capacité pour l'utilisateur de fournir l'information nécessaire (production de gestes de sélection) et pour le système de la capter (périphériques d'entrée adaptés). Dans les deux cas, les limitations sont essentiellement du côté humain.

Ainsi, les travaux de Miller [24] sur les tâches de jugement absolu, décrites comme "la précision avec laquelle un utilisateur peut assigner une valeur à la magnitude d'un ou plusieurs aspects d'un stimulus" ont montré que dans le cas d'un stimulus unidimensionnel, l'être humain est capable de distinguer de 3 à 15 catégories selon le type de stimulus (visuel, auditif, tactile, gustatif, etc.), avec une moyenne de 6.5 catégories (2.6 bits). Ce résultat est connu comme le "nombre magique $7 \pm 2$ " et a conduit à diverses recommandations sur la capacité des menus.

Cependant Miller a aussi montré que ce seuil pouvait être largement dépassé. D'une part en passant à plusieurs dimensions, c'est-à-dire en utilisant des stimuli multiples en parallèle, comme la couleur et la position 2D d'un objet (3 dimensions), ou la hauteur, le timbre et la localisation 3D d'un son (5 dimensions). Même si les capacités de chaque canal ne s'ajoutent pas, il devient possible de distinguer jusqu'à plus de 100 catégories. On peut voir l'application de cette propriété aux menus avec l'utilisation de groupements visuels, comme les sections d'un menu linéaire délimitées par un séparateur ou le placement des items de certains menus circulaires.

D'autre part Miller montre que l'on peut accroître la capacité en présentant des stimuli de façon séquentielle et en exploitant les propriétés de la mémoire, en particulier le recodage qui permet de regrouper des séquences d'information en chunks, selon un processus d'abstraction. On peut là aussi voir une application de cette propriété aux menus avec l'organisation hiérarchique qui nécessite une séquence d'interactions et l'apprentissage de gestes de sélection qui deviennent de véritables chunks [8], par exemple dans les Marking Menus.

Un autre aspect, bien connu, de l'approche informationnelle en IHM est celui des lois du mouvement humain. Ainsi la loi de Fitts [13] exprime l'indice de difficulté de la tâche en bits, et un pointage peut être interprété comme la quantité d'information que l'utilisateur fournit au système pour sélectionner une cible particulière. Comme dans les travaux de Miller, les capacités des canaux sont très faibles, de l'ordre de quelques bits par seconde dans le cas de la loi de Fitts, mais elles peuvent être accrues par le parallélisme et la séquentialité. Le parallélisme correspond aux tâche intégrales [17], qui permettent à un utilisateur de spécifier des valeurs selon plusieurs dimensions simultanément, comme par exemple un pointage en 2 ou 3 dimensions. Il est encore peu exploité dans les menus, qui organisent en général chaque niveau selon une seule dimension. La séquentialité consiste quant à elle à découper l'interaction en plusieurs étapes, et est employée pour tous les menus hiérarchiques.

Cette analyse informationnelle nous fournit un guide pour l'analyse morphologique de l'interaction avec les menus. D'une part elle nous incite à identifier les différentes $d i$ mensions d'entrée qui peuvent être mises en jeux pour la sélection d'un item. D'autre part elle nous encourage à décrire les interactions de sélection en mettant en évidence la séquentialité et le parallélisme. Enfin, elle nous conduit a analyser la manière dont une structure d'items peut être installée dans un menu.

\section{Dimensions d'entrée}

Une technique de menu peut se caractériser au niveau moteur par les canaux qu'elle prend en entrée et les caractéristiques de ces canaux. Nous définissons une dimension d'entrée selon trois facteurs :

Nature de l'information captée. Toute caractéristique mesurable pouvant être émise par un utilisateur : orientation, pression, position, courbure, nombre de doigts utilisés, hauteur de la voix, etc.;

Quantité d'information captée. À chaque étape de l'interaction, l'information captée peut-être :

- ponctuelle : une seule information est captée, même si celle-ci résulte de l'analyse d'un ensemble de données élémentaires; par exemple, l'orientation d'un trait nécessite l'analyse de la marque effectuée mais constitue une seule information;

- séquentielle : une séquence d'information est captée au fur et à mesure de l'interaction; par exemple, le Pressure Menu [25] utilise le profil de variation de la pression pour déterminer l'item sélectionné ;

Référentiel d'interprétation. Les informations transmises par une dimension d'entrée peuvent être interprétées de façon :

- absolue : Les valeurs sont interprétées dans un même référentiel, fixe et indépendant de la séquence d'interaction. Par exemple, un marking menu reconnaît les orientations cardinales et ne prend pas en compte l'orientation de la marque précédente ou la position de la marque à l'écran ;

- relative : Les valeurs sont interprétées dans un référentiel défini par la séquence d'interaction, et 
éventuellement variable. Par exemple, la sélection dans un menu contextuel classique correspond à une tâche de pointage dans un ensemble d'items dont la position dépend de l'endroit où le menu a été invoqué, qui devient l'origine du référentiel.

Un même canal peut fournir plusieurs dimensions. Par exemple les tracés d'une souris peuvent être interprétés comme deux dimensions : l'orientation et la courbure de la marque. On prend en compte ici les notions de recodage de Miller [24] ou de chunking de Buxton [8], selon lesquelles l'apprentissage et la mémorisation conduisent à représenter l'information à un plus haut niveau d'abstraction. Ainsi, avec un Flower Menu, l'utilisateur aura internalisé le fait que les deux dimensions pertinentes sont l'orientation et la courbure finale, et non pas le détail de la trajectoire de chaque geste possible.

Pour une technique donnée nous définissons la capacité d'une dimension comme le nombre maximum de valeurs reconnues par cette technique pour cette dimension. Cette capacité peut varier selon les techniques : Hybrid Menu [16] reconnaît trois valeurs de courbure là où Flower Menu [6] en autorise sept. Ces capacités sont en général issues d'observations contrôlées.

Enfin, la spécification de plusieurs dimensions peut se faire simultanément, comme l'orientation et la pression du Pressure Menu ou successivement, comme dans le cas du Zone Menu : l'utilisateur spécifie d'abord la position du sous-menu puis l'orientation correspondant à l'item visé. Dans le cas de la spécification simultanée, il faut s'assurer que les dimensions utilisées sont intégrales [17], sinon l'utilisateur ne pourra pas tirer parti du parallélisme.

\section{Interactions de sélection}

Nous représentons l'interaction de sélection d'une technique de menu dans une table dont les lignes représentent la nature et le référentiel de chaque dimension d'entrée et les colonnes la quantité d'information captée (Fig. 11). Pour chaque technique :

- Un cercle représente la saisie d'une dimension d'entrée. La saisie est obligatoire si le cercle est plein, optionnelle s'il est vide. Le nombre inscrit dans le cercle représente la capacité de la dimension considérée. Un cercle à double trait représente l'action initiale (il peut y en avoir plusieurs dans le cas d'actions simultanées).

- Une flèche représente un enchaînement d'actions successives, un trait double des actions à effectuer simultanément. L'enchaînement est obligatoire si le trait est plein, optionnel s'il est pointillé (par exemple, la progression dans une hiérarchie).

Ainsi, la sélection dans un menu linéaire hiérarchique (Fig. 11a) consiste à (i) effectuer une tâche de pointage dans un ensemble d'items empilés verticalement puis, si l'item atteint correspond à un sous-menu, (ii) traverser le bord latéral du menu parent pour sélectionner le sous-menu et retourner à l'étape (i). La tâche de pointage spécifie une

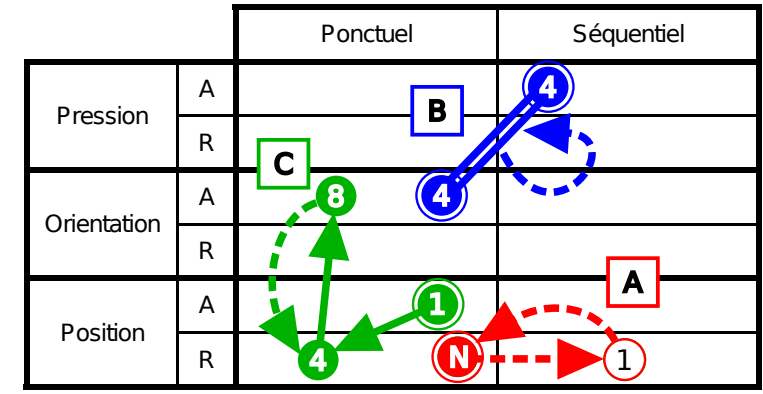

FIG. 1 : Représentation de trois techniques de menu dans notre taxonomie : menu linéaire hiérarchique (A), Pressure Menu (B) et Zone Menu (C).

position relative par rapport au point où a été appelé le menu contextuel, donc l'information de position est ponctuelle et relative, et l'ensemble de valeurs est arbitrairement grand, noté $N$. Le passage dans un sous-menu est une tâche de franchissement : le curseur doit traverser le bord du menu parent dans l'intervalle vertical correspondant à la hauteur de l'item, soit une séquence de positions relatives au sous-menu sélectionné ; cette action est binaire (soit l'utilisateur traverse la bordure et continue sa sélection, soit il reste dans le sous-menu courant) et correspond à une confirmation, elle a donc une capacité de 1. Dans un menu linéaire classique, ces tâches sont effectuées l'une après l'autre, les dimensions sont donc spécifiées en séquence.

La sélection dans un Pressure Menu [25] (Fig. 1p) consiste à spécifier une orientation et une évolution de la pression en un même geste. Les auteurs ont utilisé quatre orientations et un profil formé de la combinaison de deux valeurs, fort ou faible, au début et à la fin du geste. Nous considérons donc que la spécification de la pression est absolue et qu'elle peut prendre quatre valeurs. L'orientation et la pression étant deux composantes simultanées du geste effectué par l'utilisateur, nous considérons que leur spécification se fait en parallèle. Les auteurs évoquent une hiérarchie, que nous avons considéré comme la répétition de gestes de sélection, c'est pourquoi la flèche pointillée fait une boucle sur le trait double représentant les spécifications en parallèle.

Le Zone Menu [28] (Fig. 11.) est une technique dite tapand-stroke dans laquelle une sélection a trois étapes :

1. le tap initial définit la position du centre du menu et ainsi la position des Marking Menus; il s'agit d'une position ponctuelle absolue ;

2. la position de la marque par rapport à celle du point initial définit le Marking Menu dans lequel va se faire la sélection; c'est donc une position relative; les auteurs prévoient un maximum de quatre zones ;

3. l'orientation de la marque définit l'item sélectionné, comme pour un Marking Menu classique (capacité 8); comme pour un menu linéaire, sa valeur est spécifiée de manière absolue et ponctuelle. 


\section{Structure des menus}

Le dernier aspect de la morphologie des menus est leur structure, qui détermine la façon dont leurs items sont organisés. Cette structure est souvent hiérarchique (Fig.2p), mais elle peut aussi contenir des subdivisions, ou groupements sémantiques d'items : items "couper-copier-coller", items opposés tels que "défaire-refaire", etc.

La structure d'un menu est généralement contrainte. En effet, pour chaque dimension d'une technique, le nombre de valeurs utilisables est souvent limité : 8 orientations pour Marking Menu, 7 courbures pour Flower Menu, 4 signatures de pression pour Pressure Menu, etc. Lorsque l'on combine plusieurs dimensions, la capacité maximale est le produit de leurs capacités : $4 \times 8=32$ items pour Zone Menu, $8 \times 2=16$ items pour Polygon Menu. Ainsi, en associant une subdivision à une dimension, on peut espérer réduire la charge cognitive car l'utilisateur peut ainsi donner un sens à plusieurs caractéristiques motrices

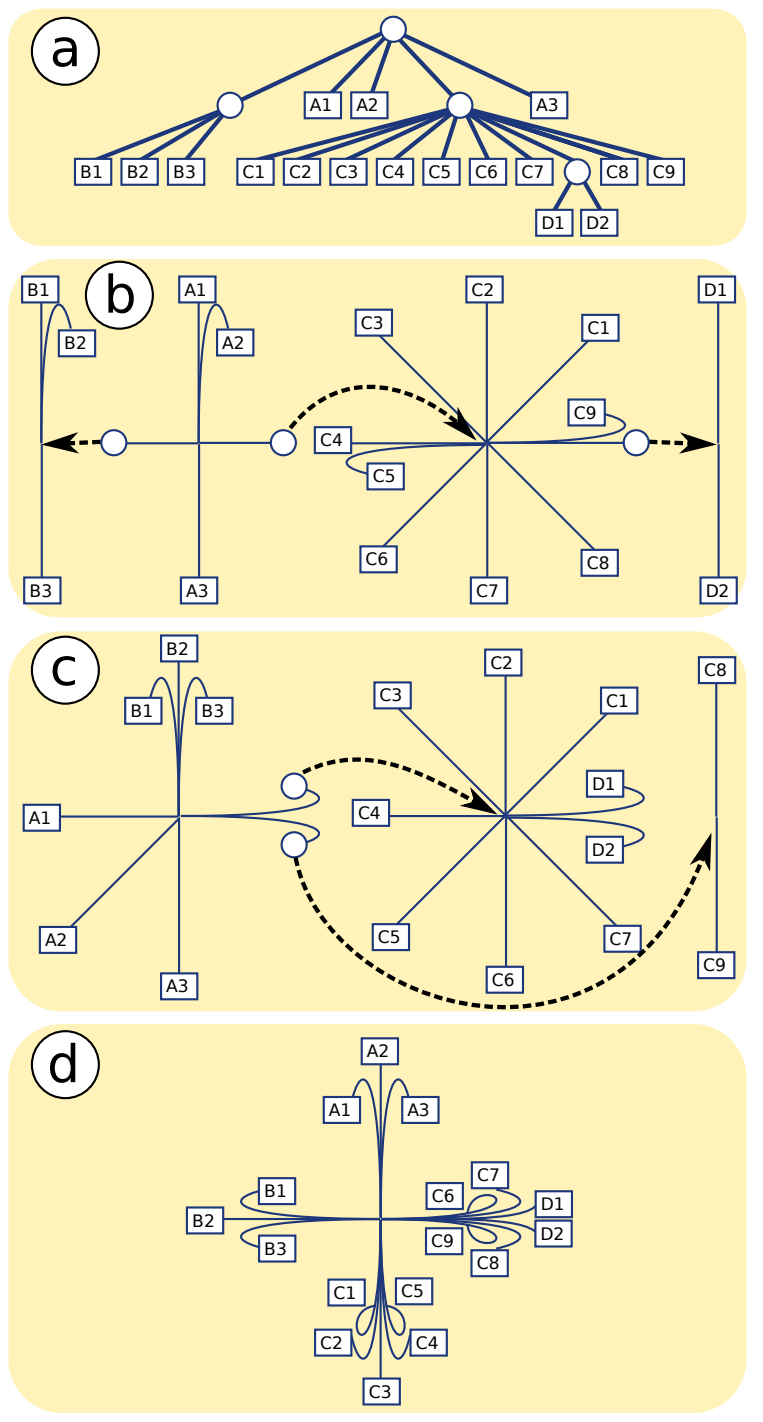

FIG. 2 : Flexibilité d'un Flower Menu. de son geste. Cependant cela introduit des contraintes de structuration supplémentaires car (a) la capacité d'une subdivision est inférieure à la capacité totale du sousmenu qui la contient et (b) certains items peuvent être au même niveau hiérarchique que d'autres subdivisions.

La figure 2 illustre différentes solutions pour une structure donnée (Fig 2a) dans un Flower Menu et démontre la flexibilité importante de ce menu, grâce à sa grande capacité et à l'utilisation de plusieurs dimensions :

- La figure 2p représente un remplissage calqué sur la structure hiérarchique. Le sous-menu le plus rempli contient 10 items (5 en moyenne) et les items les plus profonds sont au $3^{e}$ niveau hiérarchique (profondeur moyenne 2.06);

- La figure 20 représente un remplissage dans lequel l'orientation est associée aux groupes d'items. Sa largeur moyenne est de 6.33 items et sa profondeur moyenne de 1.65 niveaux ;

- La figure $2 \mathrm{~d}$ représente un remplissage favorisant la profondeur et la combinaison des dimensions. Chaque item est accessible en une seule sélection, mais la largeur du menu est de 17 items.

A titre de comparaison, un Marking menu hiérarchique imposerait une seule structure, à trois niveaux, et nécessiterait de répartir les commandes $C_{i}$ en deux sous-menus à cause de la capacité maximale de 8 items par niveau.

\section{ANALYSE DE MENUS EXISTANTS}

Nous présentons maintenant l'analyse d'un ensemble de techniques de menus selon l'approche présentée ci-dessus, en nous concentrant sur la description des dimensions et de l'interaction et en laissant de côté la structure. Nous pourrions décrire l'interaction avec des graphes CIS [2], cependant nous préférons introduire une notation inspirée des expressions régulières qui permet de combiner l'indication des dimensions et de l'interaction de façon concise.

Chaque dimension est notée Nature $_{Q, R}^{C}$ avec

- $\mathrm{Q} \in\{$ Ponctuel $(P)$, Séquentiel $(S)\}$

$-\mathrm{R} \in\{\operatorname{Absolu}(A), \operatorname{Relatif}(R)\}$

- $\mathrm{C} \in[1, \infty[$ capacité maximale de cette dimension (ou "?" si cette limite n'est pas déterminée).

Une expression régulière décrit la séquence d'interaction, c'est-à-dire la façon dont les dimensions sont spécifiées. Les opérateurs utilisés sont les suivants :

- A ? (option) : zéro ou une fois la dimension A :

- A* (répétition) : zéro ou plusieurs fois la dimension A ;

- A+ (itération) : au moins une fois la dimension A;

- A B (séquence) : dimension A puis dimension B;

- A $\mid$ B (alternative) : dimension A ou dimension B ;

- A // B (parallélisme) : les dimensions A et B sont spécifiées simultanément.

Marking Menus hiérarchiques Ces menus utilisent jusqu'à 8 valeurs d'orientation ponctuelles et absolues. Une sélection hiérarchique consiste à pratiquer plusieurs traits : Orientation ${ }_{P, A}^{8}+$ 
Flower Menu Le Flower Menu [6] multiplie par 7 la capacité d'un Marking Menu classique en ajoutant une courbure optionnelle à la fin du geste. Il y a trois valeurs de courbure, d'un côté ou de l'autre du geste orienté :

$$
\left.\begin{array}{c}
\text { Orientation }_{P, A}^{8}\left(\text { Courbure }_{P, A}^{3} / /\right. \\
\text { Sens de rotation } \\
P, A
\end{array}\right) ?
$$

Bailly et al. n'ont pas spécifié le détail d'un Flower Menu hiérarchique, mais si l'on suppose que l'utilisateur doit répéter le même type de geste à chaque niveau, on obtient :

$$
\begin{aligned}
& \text { (Orientation }_{P, A}^{8} \text { (Courbure }{ }_{P, A}^{3} / / \\
& \text { Sens de rotation } \left.{ }_{P, A}^{2}\right) \text { ?) + }
\end{aligned}
$$

Tilt Menu Le Tilt Menu [27] utilise l'altitude et l'azimuth d'un stylet (Fig. 3) pour spécifier un item. Ces canaux d'entrée sont spécifiées de manière absolue et en parallèle, car le geste permettant de spécifier l'altitude (une valeur possible) fournit une valeur d'azimuth (huit valeurs possibles). Les auteurs n'ont pas précisé la manière de rendre ce menu hiérarchique, nous nous contentons de ne représenter qu'un niveau :

$$
\text { Altitude }_{P, A}^{1} / / \text { Azimuth }_{P, A}^{8}
$$

FIG. 3 : Les canaux d'entrée utilisés par le Tilt Menu.

Zone Menu La sélection dans un Zone menu [28] commence par un tap qui spécifie l'origine d'un référentiel pour la suite de l'interaction, qui consiste en une information de position relative au tap (le début de la marque), puis une orientation absolue :

$$
\text { Position }_{P, A}^{1} \text { Position }_{P, R}^{4} \text { Orientation }_{P, A}^{8}
$$

Pour un Zone Menu hiérarchique, les marques successives sont relatives au tap initial, cependant la position du point initial de chaque marque est significative, ce qui conduit à l'interaction suivante :

$$
\text { Position }_{P, A}^{1}\left(\text { Position }_{P, R}^{4} \text { Orientation }_{P, A}^{8}\right)+
$$

Menu linéaire hiérarchique Nous avons détaillé plus haut cette interaction, qui consiste à effectuer une tâche de pointage puis à répéter les tâches de franchissement et de pointage jusqu'à ce que l'item visé soit atteint :

$$
\operatorname{Position}_{P, R}^{N}\left(\text { Position }_{S, R}^{1} \text { Position }_{P, R}^{N}\right) *
$$

HotBox La HotBox [22], grâce à sa grande capacité, est destinée à permettre un accès rapide aux nombreuses commandes d'un logiciel. L'utilisateur appuie sur la touche Espace, ce qui fait apparaître l'ensemble des menus du logiciel (Fichier, Edition, etc.) :

$$
\text { (1) Touche } e_{P, A}^{1}
$$

L'utilisateur peut alors sélectionner une commande dans ces menus linéaires, qui sont hiérarchiques, ou bien utiliser l'un des cinq Marking Menus paramétrables situés

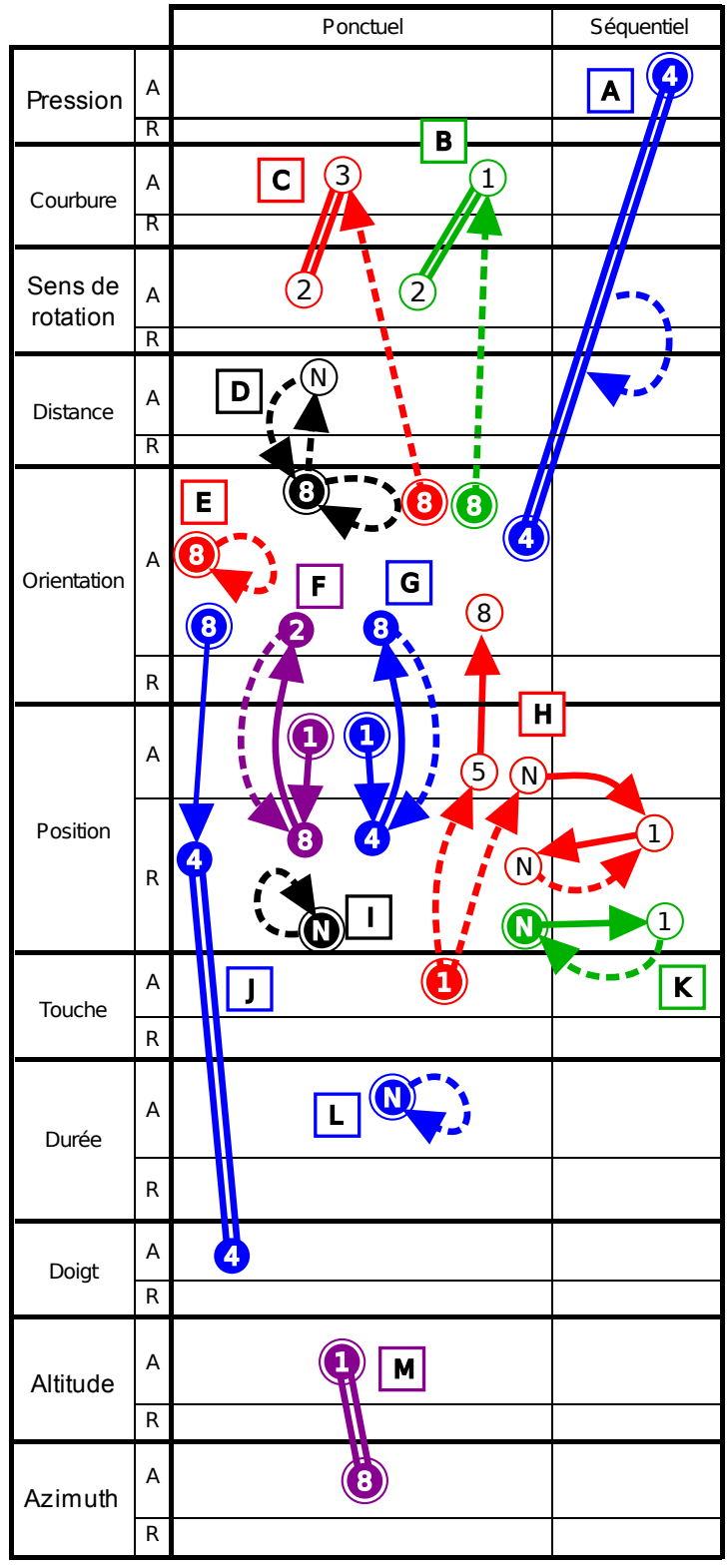

FIG. 4 : Visualisation de treize techniques de menus. A : Pressure Menu [25], B : Hybrid Menu non hiérarchique [16], C : Flower Menu non hiérarchique [6], D : Marking Menu avec sous-menus linéaires [19], E : Marking Menu hiérarchique [20], F : Polygon Menu [28], G : Zone Menu [28], H : Hotbox [22], I : Jumping Menu [1], J : MultiTouch Menu [4], K : Menu linéaire classique, L : Rythmic Menu [23], M : Tilt Menu [27]

autour du centre de l'écran. La sélection dans les menus linéaires est un peu différente de celle décrite plus haut car leur position initiale est fixe par rapport au référentiel de l'écran; la première tâche de pointage est donc absolue :

$$
\text { (2a) Position } \text { Pr,A }^{N}\left(\text { Position }_{S, R}^{1} \text { Position }_{P, R}^{N}\right) *
$$

La sélection dans les Marking Menus correspond au choix d'une zone parmi les cinq et au tracé d'une marque. Les positions de ces zones sont constantes dans le référentiel de l'écran, elle est donc absolue :

(2b) Position ${ }_{P, A}^{5}$ Orientation $_{P, A}^{8}$ 
Ces Marking Menus ne sont pas hiérarchiques, ils sont destinés à être personnalisés par les utilisateurs. L'interaction complète correspond à (1) ( (2a) | (2b)), soit :

(3) Touche $_{P, A}^{1}$ ( ( Position ${ }_{P, A}^{N}$ (Position $_{S, R}^{1}$ $\left.\left.\operatorname{Position~}_{P, R}^{N}\right) *\right) \mid\left(\right.$ Position $_{P, A}^{5}$ Orientation $\left.\left._{P, A}^{8}\right)\right)$

La figure 4 présente les techniques ci-dessus ainsi que d'autres issues de la littérature [5]. On note un usage important de la position et de l'orientation, et un faible usage des dimensions relatives et séquentielles. Ce tableau fournit donc des pistes pour explorer de nouvelles techniques.

\section{CONCEPTION DE NOUVEAUX MENUS}

L'un des objectifs de l'analyse morphologique est de structurer et faciliter l'exploration des dimensions d'entrée utilisables dans les techniques de menus afin d'augmenter leur capacité ou de les adapter à des contextes d'usage différents, tout en maintenant une relative facilité d'utilisation. Cette exploration peut consister à ajouter des dimensions, à substituer une dimension ponctuelle par une dimension séquentielle, ou inversement, à introduire du parallélisme, etc. Nous illustrons maintenant cet aspect génératif en décrivant quatre techniques inspirées par l'exploration des espaces "vides" de notre espace morphologique. Les deux premières techniques ont fait l'objet de prototypes et d'évaluations afin de valider leur faisabilité.

\section{Augmenter les Marking Menus avec la Pression}

L'utilisation de la pression pour augmenter les Marking Menu a déjà été évoquée par Ramos et al. [25] en combinant Pressure Marks (gestes prenant en compte la pression de départ et la pression finale) et Marking Menus (gestes orientés). Comme illustré Fig. 4.A, cette technique se caractérise par l'interaction (Orientation ${ }_{P, A}^{4} / /$ Pression $\left._{S, A}^{4}\right)+$ : en parallèle à l'orientation ponctuelle, la pression est utilisée de manière séquentielle, en prenant en compte son évolution entre deux seuils absolus (faible et fort). Cette technique n'a pas été implémentée ni évaluée par les auteurs.

Une manière alternative d'ajouter la pression à un Marking Menu est d'utiliser une pression ponctuelle plutôt que séquentielle. Nous avons donc imaginé PushMenu, qui associe une pression ponctuelle à un Marking Menu standard : $\left(\right.$ Orientation ${ }_{P, A}^{8} / /$ Pression $\left._{P, A}^{?}\right)+$.

L'utilisation de la pression comme dimension ponctuelle permet de concevoir un retour visuel proche d'un Marking Menu, où tous les items du menu sont visibles simultanément pour chaque niveau de pression. La dimension "orientation" est représentée comme dans un menu circulaire classique, par des secteurs angulaires. Pour représenter le niveau de pression à atteindre, les items sont regroupés en anneaux concentriques, le niveau de pression le plus faible correspondant à l'anneau le plus éloigné (Fig. 5). Au fur et à mesure que la pression exercée par l'utilisateur augmente, la taille de l'anneau actif diminue et celle de l'anneau représentant le seuil de pression sui-

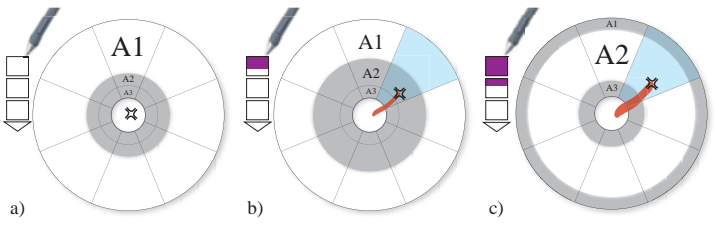

FIG. 5 : Evolution de la représentation visuelle d'un PushMenu à 3 niveaux en fonction de la pression.

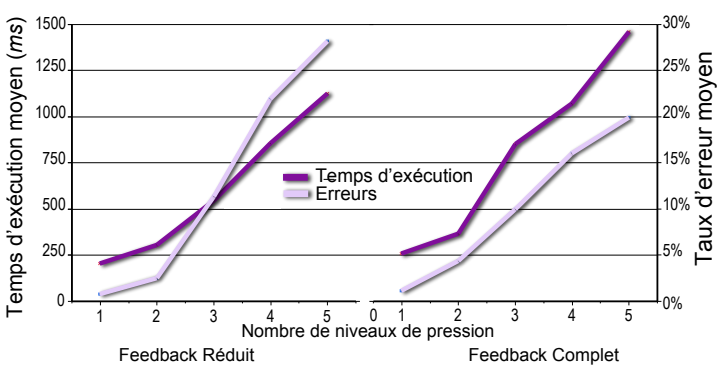

FIG. 6 : PushMenu : temps d'exécution et taux d'erreur selon le nombre de niveaux de pression $(\mathrm{N})$ et les conditions de feedback $(\mathrm{F})$.

vant augmente (Fig. 5.b). Lorsque la pression franchit le seuil entre deux anneaux, le nouvel anneau actif est mis en évidence par sa couleur différente (Fig. 5-c). Ainsi, la sélection d'un item se fait selon la direction du geste et le niveau de pression final, que ce soit en mode novice ou expert (avec ou sans retour visuel).

Afin de valider cette technique, nous avons mené une expérimentation contrôlée pour vérifier que la spécification simultanée de l'orientation et de la pression est possible (dimensions intégrales), et pour déterminer le nombre de niveaux de pression utilisables de manière fiable et efficace. Le protocole consistait à sélectionner des items dans des PushMenu ayant de 1 (marking menu) à 5 niveaux de pression, en mode novice (avec retour graphique) ou expert (seule une marque était affichée).

L'analyse des données (10 participants, 7200 sélections) nous a fourni les principaux résultats suivants (Fig. 6). Comme pour le Marking Menu classique, les performances en temps d'exécution sont meilleures sans feedback. Le temps d'exécution et le taux d'erreurs pour les niveaux 1 et 2 ne sont pas significativement différents, ce qui indique qu'un PushMenu a des performances équivalentes à un Marking Menu jusqu'à une capacité de 16 items. Enfin, les participants ont su discriminer jusqu'à trois niveaux de pression avec moins de $10 \%$ d'erreur. Ces résultats permettent d'envisager l'utilisation de PushMenus de capacité $8 \times 3=24$ items, avec deux possibilités de groupement : 8 groupes de 3 items ou 3 groupes de 8 .

En conclusion, cette technique utilise une combinaison de dimensions qui n'avait pas été explorée jusqu'à présent et que l'espace de conception nous a permis d'identifier. Même si la capacité théorique (24 items) est moindre que celle du Pressure Menu (32 items), nous pensons que sa 


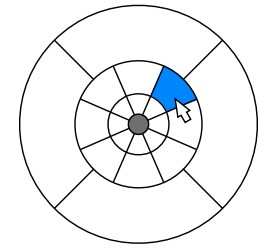

FIG. 7 : Principe du Dartboard Menu.

plus grande similitude avec le Marking Menu classique et son mode novice plus simple à représenter en font une alternative intéressante au Pressure Menu.

\section{Augmenter les Marking Menus avec la Distance}

En suivant la même démarche que pour la pression, nous avons étudié l'utilisation de la distance comme dimension supplémentaire aux Marking Menus pour augmenter leur capacité sans perdre leurs propriétés fondamentales (transition du mode novice au mode expert et simplicité des gestes à réaliser). Le principe est un Marking Menu dont chaque niveau est constitué de plusieurs anneaux concentriques (Fig. 7), que l'on peut donc caractériser ainsi : (Orientation ${ }_{P, A}^{8} / /$ Distance $\left._{P, A}^{?}\right)+$. Un tel menu est mentionné dans la littérature par Kurtenbach [18], qui l'appelle Dartboard mais ne l'étudie pas plus avant. Le Bullseye Menu [15] est similaire, mais est conçu pour des utilisateurs malvoyants, avec un feedback sonore. Nous avons conduit deux études pour valider ce menu.

Expérience 1 : caractérisation des longueurs. Cette étude a pour but de définir le nombre de longueurs différentes qu'un utilisateur peut produire de façon fiable dans le mode expert du Dartboard, et de caractériser l'espace des longueurs optimal, c'est-à-dire les rayons des anneaux successifs minimisant les erreurs de sélection. Huit volontaires ont tracé des traits rectilignes suivant les huit orientations cardinales, avec pour consigne de donner à leur tracé une longueur de " $i$ sur un total de $N$ ", avec $N \in[2 ; 5]$ et $i \in[1 ; N]$. L'objectif était de ne donner qu'une information relative sur la longueur des traits afin d'observer la manière dont les utilisateurs découpent l'espace de longueurs. Il s'agit en quelque sorte d'une expérience inverse des expériences de Miller [24] : il ne s'agit pas de juger la magnitude des stimuli mais de produire des stimuli dont la magnitude est contrôlée.

Le premier résultat de cette étude est que le contrôle de la longueur n'ajoute pas de difficulté aux mécanismes de contrôle du Marking Menu et que ces dimensions sont donc intégrales. En effet, les participants ont fait moins de $2 \%$ d'erreurs d'orientation au total, et $2 \%$ de traits non linéaires en moyenne. Nous avons ensuite déterminé des espaces de longueurs en calculant, pour chaque valeur de $N$, les $N-1$ frontières permettant de maximiser le nombre d'essais "réussis", c'est-à-dire ceux dont la magnitude relative à ces frontières correspond à la consigne (Fig. 8). Ces calculs montrent qu'au-delà de 4 niveaux,

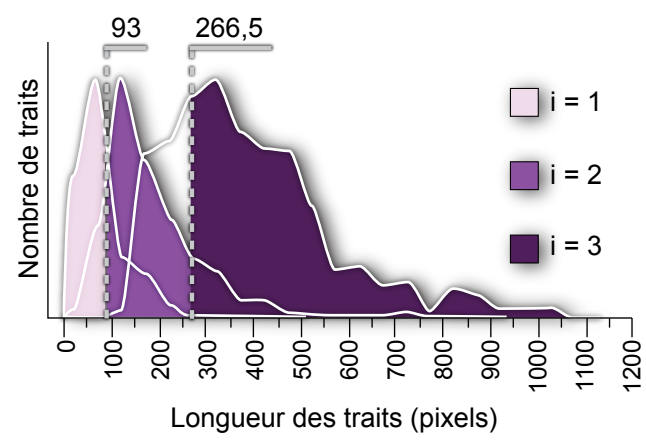

FIG. 8: Espaces de longueur du Dartboard menu $(\mathrm{N}=3)$.

la différenciation des longueurs est peu fiable $(<70 \%)$. C'est pourquoi nous avons considéré pour la suite $\mathrm{N}=2,3$ ou 4, soit des menus pouvant contenir 16, 24 ou 32 items, soit (Orientation ${ }_{P, A}^{8} / /$ Distance $\left._{P, A}^{4}\right)+$.

Expérience 2 : comparaison des performances La seconde expérience a comparé les performances du mode expert du Dartboard Menu avec Zone Menu [28] et Flower Menu [6]. Ces deux techniques sont particulièrement intéressantes car elles augmentent également la capacité du Marking Menu par l'ajout d'une nouvelle dimension : Position $_{P, A}^{4}$ Orientation $_{P, A}^{8}$ pour Zone Menu et $\left(\right.$ Courbure $_{P, A}^{4} / /$ Sens $\left._{P, A}^{2}\right)$ ? pour Flower Menu.

12 sujets ont effectué des sélections parmi 16, 24 et 32 items sur une tablette-écran avec chacune des trois techniques, soit 7776 sélections. Nos analyses montrent que Dartboard est plus rapide que Flower Menu, qui est plus rapide que Zone Menu. Cependant, le temps de réaction de Zone Menu est plus court que Flower Menu et Dartboard, et le taux d'erreur du Flower Menu est plus élevé que celui de Dartboard, lui-même plus élevé que celui de Zone Menu. Ces résultats peuvent s'expliquer par le fait que le contrôle de la courbure et de la longueur ne sont pas des tâches habituelles et demandent un temps de préparation plus long. Le temps de sélection d'un item parmi 16 est plus court qu'une sélection parmi 24 items, lui-même plus court qu'une sélection parmi 32 items. Ce résultat est intéressant car, pour chaque technique, le nombre de gestes est indépendant du nombre d'items. Enfin, Dartboard provoque moins de $10 \%$ d'erreurs avec 2 ou 3 anneaux, soit une capacité de 16 ou 24 items.

Ces expériences montrent que Dartboard permet d'augmenter la capacité d'un Marking Menu. Il n'est ni globalement meilleur ni globalement moins bon que les deux autres techniques testées. Cela renforce l'idée que la performance limite lorsque l'on augmente le nombre de dimensions (distance pour Dartboard, courbure pour Flower Menu, position relative pour Zone Menu) pourrait être relativement indépendante des dimensions mises en jeu. 


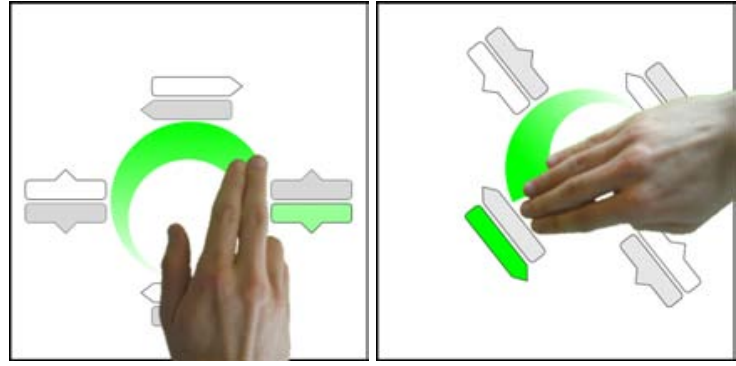

FIG. 9 : Dial Menu, un menu utilisant la configuration des doigts, la courbure du geste et son sens de rotation.

\section{Exploiter l'azimuth dans les interfaces à stylet}

Les canaux d'entrée 'altitude' et 'azimuth' ont été utilisés dans la technique du Tilt Menu [27] pour permettre la sélection d'items sans interférence avec la tâche réalisée avec la pointe du stylet (pointage ou tracé). Nous proposons d'utiliser cette dimension 'azimuth' avec des Marking Menus pour en augmenter leur capacité. Sélectionner un item avec cet AziMenu reviendrait alors à tracer un geste avec la pointe du stylet dans la direction voulue (la dimension Orientation du Marking Menu standard) tout en donnant une orientation particulière au stylet (la dimension Azimuth), soit : (Azimuth? ${ }_{P, A}^{?} / /$ Orientation $\left._{P, A}^{8}\right)+$ pour un AziMenu hiérarchique. En considérant les limites déjà identifiées pour la dimension Orientation dans les menus circulaires ( 8 orientations), et en supposant que le contrôle de l'azimuth permette de distinguer quatre valeurs, on atteint une capacité théorique de $8 \times 4$ items par sous-menu (ou 8 groupes de 4 ou 4 groupes de 8 selon la dimension associée aux subdivisions).

Outre qu'elle permet d'augmenter la capacité d'un Marking Menu, cette approche peut, comme le Tilt Menu, discriminer entre un mode tracé et un mode commande dans les interfaces à stylet : il suffit de réserver certaines valeurs de l'azimuth au tracé et de consacrer les autres aux menus. Bien entendu, l'utilisabilité de l'AziMenu dépend du bon contrôle de la dimension supplémentaire, notamment l'effet des rotations non intentionnelles du stylet, et nécessite de déterminer expérimentalement les limites de cette dimension dans les modes novice et expert.

\section{Un menu indépendant de l'orientation pour les tables}

L'utilisation des doigts comme canal d'entrée a déjå été proposée dans le MultiTouch Menu [4]. Dans notre espace de conception, nous pouvons considérer ce canal de manière relative (la valeur prise en compte est le nombre de doigts utilisés) ou absolue (quels doigts sont utilisés). Toutefois, la plupart des dispositifs multitouch ne permettent pas d'identifier quel doigt est posé, ce qui élimine la dimension absolue. Par ailleurs, la courbure et le sens de rotation ont été utilisés avec le Flower Menu [6]. Il a entre autre été montré qu'un utilisateur pouvait facilement différencier les deux sens de rotation et jusqu'à 4 courbures différentes sans retour visuel.

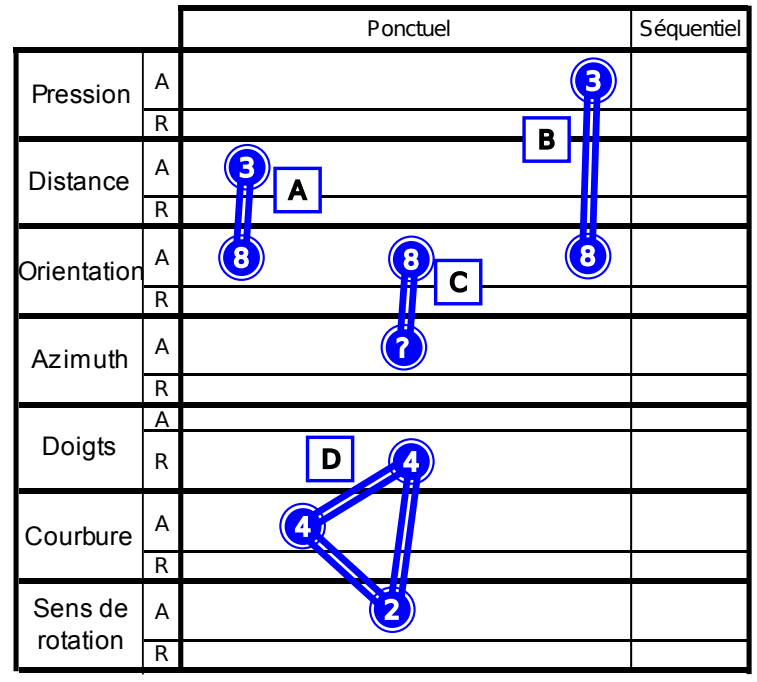

FIG. 10 : Les 4 techniques proposées : Dartboard (A), Push Menu (B), AziMenu (C) et Dial Menu (D)

Nous proposons d'associer ces 3 dimensions en parallèle dans un Dial Menu décrit par : (Doigts ${ }_{P, R}^{4} / /$ Courbure $_{P, A}^{4}$ // Sens de rotation $\left._{P, A}^{2}\right)+$. La sélection d'un item consiste à tracer des portions de cercle avec un à quatre doigts (Fig. 9). Les items sont représentés par paire, un par sens de rotation, ceux correspondant au sens de rotation non actif sont grisés et l'item sélectionné est en vert.

Dial Menu parait bien adapté à l'utilisation collaborative d'une table tactile, dans laquelle l'orientation absolue est source de problèmes. Il offre une capacité maximale de 4 (doigts) $\times 4$ (courbure) $\times 2($ sens de rotation $)=32$ items, qui pourrait être augmentée si l'on distingue d'autres combinaisons de doigts. Il permet également l'utilisation de groupes, que l'on peut associer aux doigts ou à la courbure, et d'opposés, associés au sens de rotation.

\section{CONCLUSION}

Nous avons présenté un espace de conception analytique et génératif permettant de faciliter l'exploration et l'implémentation de nouvelles techniques de menus. Cet espace est fondé sur une taxonomie des dimensions utilisées par les techniques de menu, qui permet d'analyser de manière précise la spécification d'une sélection dans un menu et de visualiser les combinaisons de dimensions non explorées. Nous avons présenté quatre exemples de menus (Fig. 10 créés à l'aide de notre espace de conception, dont deux ont été étudiées et évaluées. Nous avons montré que ces deux menus sont des alternatives acceptables aux techniques de la littérature, et ainsi que cet espace de conception permet de générer des menus utilisables.

Nous allons poursuivre ces travaux selon plusieurs directions. D'une part nous allons développer l'étude structurelle des menus afin de mieux quantifier la capacité d'un menu à organiser les items de différentes manières, et de mettre en évidence des propriétés souhaitables, comme 
les groupements ou les appariements (commandes fairedéfaire par exemple). D'autre part nous allons compléter l'approche par un pouvoir prédictif, en exploitant les outils développés pour le modèle CIS [2].

Par ailleurs nous allons étudier l'ambiguïté du formalisme : une même technique peut être analysée de différentes manières (par exemple la courbure du Flower Menu); à l'inverse une même description peut correspondre à plusieurs techniques de menus (par exemple Multistroke et Compound Menus [29]). Le but n'est pas nécessairement d'éliminer ces ambiguïtés, qui sont inhérentes à toute modélisation, mais de les caractériser. Enfin nous allons poursuivre l'exploration de l'espace de conception pour créer et évaluer de nouvelles techniques de menus.

\section{BIBLIOGRAPHIE}

1. D. Ahlstroem, R. Alexandrowicz, and M. Hitz. Improving menu interaction : a comparison of standard, force enhanced and jumping menus. In Proc. $\mathrm{CHI}$ '06, 1067-1076. ACM, 2006.

2. C. Appert. Modélisation, Évaluation et Génération de Techniques d'Interaction. $\mathrm{PhD}$ thesis, Université Paris-Sud, 2006.

3. G. Bailly. Techniques de menus : Caractérisation, Conception et Evaluation. $\mathrm{PhD}$ thesis, Université de Grenoble, 2009.

4. G. Bailly, A. Demeure, E. Lecolinet, and L. Nigay. Multitouch menu (mtm). In Proc. IHM '08, 165168. ACM, 2008.

5. G. Bailly, E. Lecolinet, and L. Nigay. Quinze ans de recherche sur les menus : Critères et propriétés des techniques de menus. In Proc. IHM'07. ACM, 2007.

6. G. Bailly, E. Lecolinet, and L. Nigay. Flower menus : a new type of marking menu with large menu breadth, within groups and efficient expert mode memorization. In Proc. AVI'08, 15-22. ACM, 2008.

7. T. Baudel. Aspects Morphologiques de l'Interaction Humain-Ordinateur : Étude de Modèles d'Interaction Gestuels. PhD thesis, Univ. Paris-Sud, 1995.

8. W. Buxton. Chunking and phrasing and the design of human-computer dialogues. In Proc. IFIP WCC, 475-480. North Holland Publishers, 1986.

9. J. Callahan, D. Hopkins, D. Hopkins, M. Weiser, and B. Shneiderman. An empirical comparison of pie vs. linear menus. In Proc. CHI'88, 95-100. ACM, 1988.

10. S. K. Card, J. D. Mackinlay, and G. G. Robertson. A morphological analysis of the design space of input devices. ACM Trans. Inf. Syst., 9(2) :99-122, 1991.

11. S. K. Card, A. Newell, and T. P. Moran. The Psychology of Human-Computer Interaction. Lawrence Erlbaum Associates, Inc., 1983.

12. A. Cockburn, C. Gutwin, and S. Greenberg. A predictive model of menu performance. In Proc. CHI'07, 627-636. ACM, 2007.
13. P. M. Fitts. The information capacity of the human motor system in controlling the amplitude of movement. J. Exper. Psych., 47 :381-391, 1954.

14. J. D. Foley, V. L. Wallace, and P. Chan. The human factors of computer graphics interaction techniques. IEEE Comput. Graph. Appl., 4(11) :13-48, 1984.

15. N. Friedlander, K. Schlueter, and M. Mantei. Bullseye ! when Fitts' law doesn't fit. In Proc. CHI'98, 257-264. ACM, 1998.

16. P. Isokoski and M. Käki. Comparison of two touchpad-based methods for numeric entry. In Proc. CHI'07, 25-32. ACM, 2002.

17. R. J. K. Jacob, L. E. Sibert, D. C. Mcfarlane, and M. P. Mullen. Integrality and separability of input devices. ACM TOCHI, 1 :3-26, 1994.

18. G. Kurtenbach. The Design and Evaluation of Marking Menus. PhD thesis, Univ. of Toronto, 1993.

19. G. Kurtenbach. Display and control of menus with radial and linear portions. US Patent 5926178, 1999.

20. G. Kurtenbach and W. Buxton. The limits of expert performance using hierarchic marking menus. In Proc. INTERCHI'93, 482-487. ACM, 1993.

21. G. Kurtenbach and W. Buxton. User learning and performance with marking menus. In Proc. CHI'94, 258-264. ACM, 1994.

22. G. Kurtenbach, G. W. Fitzmaurice, R. N. Owen, and T. Baudel. The hotbox : efficient access to a large number of menu-items. In Proc. CHI'99, 231-237. ACM, 1999.

23. S. Maury, S. Athénes, and S. Chatty. Rhythmic menus : toward interaction based on rhythm. In Proc. CHI '99, 254-255. ACM, 1999.

24. G. A. Miller. The magical number seven, plus or minus two : Some limits on our capacity for processing information. Psych. Review, 63 :81-97, 1956.

25. G. A. Ramos and R. Balakrishnan. Pressure marks. In Proc. CHI'07, 1375-1384. ACM, 2007.

26. M. A. Tapia and G. Kurtenbach. Some design refinements and principles on the appearance and behavior of marking menus. In Proc UIST'95, 189-195. ACM, 1995.

27. F. Tian, L. Xu, H. Wang, X. Zhang, Y. Liu, V. Setlur, and G. Dai. Tilt menu : using the $3 \mathrm{~d}$ orientation information of pen devices to extend the selection capability of pen-based user interfaces. In Proc. CHI'08, 1371-1380. ACM, 2008.

28. S. Zhao, M. Agrawala, and K. Hinckley. Zone and polygon menus : using relative position to increase the breadth of multi-stroke marking menus. In Proc. CHI'06, 1077-1086. ACM, 2006.

29. S. Zhao and R. Balakrishnan. Simple vs. compound mark hierarchical marking menus. In Proc. UIST '04, 33-42. ACM, 2004. 